\title{
Sugar signaling is involved in histone deacetylase-mediated repression of embryonic characteristics after germination
}

\author{
Motoki Tanaka, Akira Kikuchi*, Hiroshi Kamada \\ Graduate School of Life and Environmental Sciences, University of Tsukuba, Tsukuba, Ibaragi 305-8572, Japan \\ *E-mail: kikuike@sakura.cc.tsukuba.ac.jpＴel \& Fax: +81-29-853-7729
}

Received March 12, 2008; accepted May 12, 2008 (Edited by H. Shimada)

\begin{abstract}
Sugars are important regulators of germination and seedling development, and sugar signaling is thought to be involved in the repression of embryonic characteristics after germination. In contrast, chromatin remodeling induced by histone modification plays an important role in the control of plant development. We found that HDA6 and HDA19 redundantly repress embryo-specific gene expression during germination in Arabidopsis. We analyzed the effects of sucrose-containing or sucrose-free medium on post-germination growth arrest in an HDA6/19 double repression line (HDA6/19:RNAi). Although HDA6/19:RNAi seeds showed post-germination growth arrest when grown in sucrosecontaining medium, sucrose-free medium relieved growth arrest, indicating the suppression of embryo-specific genes. Thus, sugar signaling may prevent the HDAC-mediated repression of embryonic characteristics after germination. Based on expression analyses of VSP2 in HDA6/19:RNAi seedlings, the repression of HDA6 and HDA19 may not affect sugar signaling. During germination, HDA6 and HDA19 repress embryonic properties via regulation of embryo-specific transcription factors. Sugar signaling may also contribute to this repression of embryonic properties.
\end{abstract}

Key words: Embryonic property, germination, HADC, post-germination growth arrest, sucrose.

Plant cells undergo drastic changes during seed germination. Before germination, plant cells are dormant and possess some seed reserve substances. After germination, the cells use the seed reserve substances to initiate growth and greening. During the transition from germination to post-germination growth, embryonic properties are lost as an autotrophic nutrition system is established. In multicellular organisms, the defining characteristics of each organ and each developmental stage are determined by the expression of a specific gene cluster. During germination in higher plants, the gene cluster responsible for embryonic properties is suppressed and the cells lose embryonic characters, resulting in a phase transition from embryogenesis to vegetative growth.

Chromatin remodeling induced by histone modification plays an important role in several biological phenomena in eukaryotes, and genetic studies in model plants have demonstrated the importance of chromatin remodeling factors in the control of plant development (Köhler and Grossniklaus 2002; Wanger 2003). Histonedeacetylase (HDAC) catalyzes histonedeacetylation and is involved in various phenomena associated with transcriptional repression. Several studies have examined the putative functions of HDACs in plant development (Chen and
Tian 2007). In Arabidopsis, the embryo-specific transcription factors LEAFY COTYLEDON 1 (LEC1), FUSCA3 (FUS3), and ABSCISIC ACID INSENSITIVE 3 (ABI3) play key roles in embryogenesis (To et al. 2006; Suzuki et al. 2007). Although the repression of embryogenesis-related genes during germination is thought to occur, the role of HDAC in this process has not been elucidated. In a previous study, we revealed that the treatment of Arabidopsis seeds with an HDAC inhibitor resulted in growth arrest and increased transcription of LEC1, FUS3, and ABI3 during germination with $90 \mathrm{mM}$ sucrose (Tanaka et al. 2008). Among HDAC mutants, an HDA6 repression line showed growth arrest and the expression of embryospecific transcription factors after germination in the presence of low concentrations of HDAC inhibitor. In addition, an HDA6/HDA19 double repression line (HDA6/19:RNAi) displayed post-germination growth arrest even in the absence of an HDAC inhibitor with $90 \mathrm{mM}$ sucrose (Tanaka et al. 2008).

HDAC-deficient seedlings that undergo growth arrest after germination show strong similarities to seedlings that undergo growth arrest as a result of treatment with high concentrations of sugar (Price et al. 2003).

Abbreviations: ABI3, ABSCISIC ACID INSENSITIVE 3; FUS3, FUSCA3; HDAC, histonedeacetylase; LEC1, LEAFY COTYLEDON 1; PHE1, PHRES1; VSP2, VEGETATIVE STORAGE PROTEIN 2; Ws, ecotype Wassilewskija (wild type).

This article can be found at http://www.jspcmb.jp/ 
Furthermore, the disruption of a gene involved in sugar signaling induces post-germination growth arrest in synchrony with the ectopic expression of LEC1, FUS3, and other embryogenesis-related genes; these phenotypes are dependent upon the presence of sugars (Tsukagoshi et al. 2007). Based on these results, sugar signaling is thought to be involved in the post-germination growth arrest observed in an HDA6/19:RNAi line. In this paper, we examined the contribution of sugar signaling to the repression of embryogenic properties during germination.

\section{Materials and methods}

\section{Plant materials}

The Arabidopsis thaliana ecotype Wassilewskija (Ws; the wild type), HDA6/19:RNAi-1 (CS24039/CS30925), and HDA6/ 19:RNAi-1lec1-1were examined (Tanaka et al. 2008). For germination, seeds were surface-sterilized in a sodium hypochlorite solution (with an available chlorite concentration of $1 \%$ ) for $5 \mathrm{~min}$ and then rinsed five times in sterile distilled water. The surface-sterilized seeds were incubated at $4^{\circ} \mathrm{C}$ for 4 days, sown in plastic Petri dishes $(9 \mathrm{~cm}$ diameter) containing $20 \mathrm{ml}$ of phytohormone-free Gamborg's B5 solid medium $(0.8 \%$ agar, w/v), and incubated under a $16-\mathrm{h}: 8$-h light: dark cycle at $25^{\circ} \mathrm{C}$. To analyze the effects of sucrose on postgermination growth, surface-sterilized Ws and $H D A 6 / 19$ :RNAi seeds were sown in B5 solid medium in the presence or absence of $90 \mathrm{mM}$ sucrose.

\section{$R T-P C R$ expression analysis}

Total RNA was isolated using an RNAqueous Kit (Ambion, Austin, TX, USA). cDNAs were synthesized using the SuperScript First-Strand Synthesis System for RT-PCR (Invitrogen, Carlsbad, CA, USA). Each gene was amplified under the following conditions: $94^{\circ} \mathrm{C}$ for $10 \mathrm{~min}$, followed by $25-40$ cycles of $94^{\circ} \mathrm{C}$ for $15 \mathrm{~s}, 55^{\circ} \mathrm{C}$ for $15 \mathrm{~s}$, and $72^{\circ} \mathrm{C}$ for $1 \mathrm{~min}$. The forward and reverse primers were as follows: $L E C 1$, 5'-AGACGGCAGAGAAACAATGG-3' and 5'-ATTCATCTTGACCCGACGAC-3'; VSP2, 5'-ACGCAAAATATGGATACGGA-3' and 5'-AAGGTACGTAGTAGAGTGGA-3'; UBQ10, 5'-GATCTTTGCCGGAAAACAATTGGAGGATGGT-3' and 5'-CGACTTGTCATTAGAAAGAAAGAGATAACAGG-3' .

Quantitative reverse-transcription polymerase chain reaction (RT-PCR) was performed using a LightCycler (Roche Applied Science, Indianapolis, IN, USA) and the LightCycler FastStart DNA Master SYBR Green I Kit (Roche Applied Science). PHE1 transcripts were amplified using the primers 5'-CGCATGTGCGGTCATCC-3' and 5'-TCCAACACCGAAAACTCCAT-3'. The amount of cDNA produced was calculated using LightCycler 3.1 (Roche Applied Science). PHE1 expression was normalized against that of $U B Q 10$, which was amplified using the primers $5^{\prime}$-GTACTTTGGCGGATTACAACA TC-3' and 5'-GAATACCTC CTT GTCCTGGAT CT-3'.

\section{Staining with fat red $7 B$}

Seven-day-old seedlings from the Ws and HDA6/19:RNAi lines were germinated in medium with or without $90 \mathrm{mM}$ sucrose, and then stained with a $0.1 \%(\mathrm{w} / \mathrm{v})$ solution of fat red $7 \mathrm{~B}$
(Sigma) as described by Brundrett et al. (1991). Briefly, a 0.1\% solution of fat red 7B was prepared by dissolving the dye in polyethylene glycol (average molecular mass $=400 \mathrm{D}$, Sigma) for $1 \mathrm{~h}$ at $90^{\circ} \mathrm{C}$. An equal volume of $90 \%(\mathrm{v} / \mathrm{v})$ glycerol was added. Before staining, chlorophyll was removed from seedlings by incubating overnight in $70 \%(\mathrm{v} / \mathrm{v})$ ethanol. Seedlings were stained for $1 \mathrm{~h}$ at room temperature, rinsed several times in water, and then observed under a Leica DMR microscope using bright-field Nomarski optics.

\section{Generation of PHE1::GUS transgenic plants}

The generation of a PHE $1:$ GUS transgenic line was performed as described by Köhler et al. (2003). The PHE1 promoter, contained within a 2961-bp sequence of the 5 ' flanking region, was amplified by PCR using the primers $5{ }^{\prime}$-CACCGAATTCGACTTTAAAATAGTAGAAAAGCTTG- $3^{\prime}$ and 5'-AATTCCATGGATCTCTTATCTTTTTCTTTTGTG-3'. The amplified PCR product was cloned into pENTR-D-TOPO (Invitrogen). This construct was subcloned into the binary vector pKGWFS7 (Karimiet al. 2002), positioning the PHE1 promoter upstream of the $\beta$-glucuronidase gene, and then introduced into Agrobacterium tumefaciens strain GV2260. Wild-type Ws plants were transformed via the floral dip method. PHE1::GUS plants were crossed with $H D A 6 / 19$ :RNAi plants to introduce the PHE1::GUS transgene.

\section{Staining for GUS activity}

Seven-day-old seedlings and siliques that had been longitudinally sliced were fixed for $1 \mathrm{~h}$ at $-20^{\circ} \mathrm{C}$ in $90 \%$ acetone and then washed three times in $50 \mathrm{mM}$ phosphate buffer ( $\mathrm{pH}$ 7.0). The specimens were incubated at $37^{\circ} \mathrm{C}$ in reaction buffer $(0.19 \mathrm{mM} 5$-bromo-4-chloro-3-indolyl- $\beta$-Dglucuronide, $10 \mathrm{mM}$ EDTA, $0.1 \%$ Triton X-100, $0.1 \mathrm{mM}$ $\mathrm{KFeCN}, 50 \mathrm{mM}$ phosphate buffer, $\mathrm{pH}$ 7.2) for $24 \mathrm{~h}$. Whole seeds were observed after clearing in Hoyer's medium (Grossniklaus et al. 1998) using a Leica DMR microscope under bright-field Nomarski optics. Seedlings were observed without clearing in Hoyer's medium.

\section{Results}

\section{Effects of sugar on post-germination growth arrest in an HDA6/19 double repression line}

Wild-type and HDA6/19:RNAi-1 seeds were sown in solid medium in the presence or absence of $90 \mathrm{mM}$ sucrose. In wild-type seeds, no difference was observed in the rate of germination or post-germination growth in response to sucrose. In contrast, the rate of postgermination growth in $H D A 6 / 19$ :RNAi seeds more than doubled when they were sown in sucrose-free medium (Figures 1 and 2). Because growth arrest is coupled with the continued expression of embryo-specific genes and some embryonic properties (Tanaka et al. 2008), LEC1 expression was analyzed in wild-type and $H D A 6 / 19$ : RNAi seeds germinated in medium with or without sucrose. LEC1 expression was not observed in HDA6/ 19:RNAi seedlings when they were germinated in 


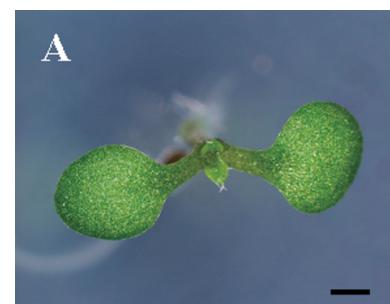

Ws +Suc

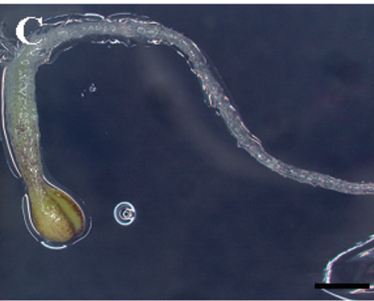

HDA6/19:RNAi-1

+ Suc

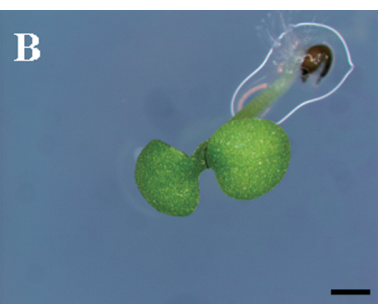

Ws -Suc

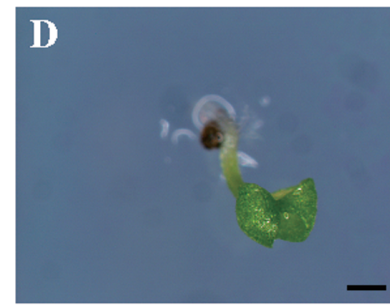

HDA6/19:RNAi-1 -Suc
Figure 1. Effect of $90 \mathrm{mM}$ sucrose on post-germination growth in wild-type (ecotype Wassilewskija, Ws) and $H D A 6 / 19$ double repression (HDA6/19:RNAi) seedlings. (A) Ws seedlings sown in sucrosecontaining medium. (B) Ws seedlings sown in sucrose-free medium. (C) HDA6/19:RNAi-1 seedlings sown in sucrose-containing medium. (D) $H D A 6 / 19:$ RNAi-1 seedlings sown in sucrose-free medium. Photographs were taken 7 days after sowing. Bar $=1 \mathrm{~mm}$.

sucrose-free medium (Figure 3). Furthermore, we used fat red 7B to confirm that these germinated seedlings had lost their embryonic properties. Because fat red 7B interacts with neutral lipid stores (Brundrett et al. 1991), positive staining indicates the presence of large amounts of triacylglycerol, an embryonic characteristic (Henderson et al. 2004). Fat red 7B staining was not observed in wild-type seedlings germinated in medium with or without sucrose (Figure 4). Dense staining was observed in the closed cotyledon and the hypocotyl of HDA6/19: RNAi seeds germinated in sucrose-containing medium, but not in the growing root (Figure 4). In contrast, staining intensity was much weaker in HDA6/19:RNAi seedlings germinated in sucrose-free medium.

To examine whether the repression of HDA6 and $H D A 19$ was involved in sucrose signaling, we examined the expression of VEGETATIVE STORAGE PROTEIN 2 (VSP2), a sugar-inducible gene (Dejardin et al. 1999). $V S P 2$ expression did not differ between wild-type and HDA6/19:RNAi seeds germinated in medium with or without $90 \mathrm{mM}$ sucrose (Figure 3).

\section{Contribution of HDA6 and HDA19 to the regulation of PHE1 expression during embryogenesis}

We also analyzed the expression of another embryospecific gene, PHRES1 (PHE1), which encodes a
A

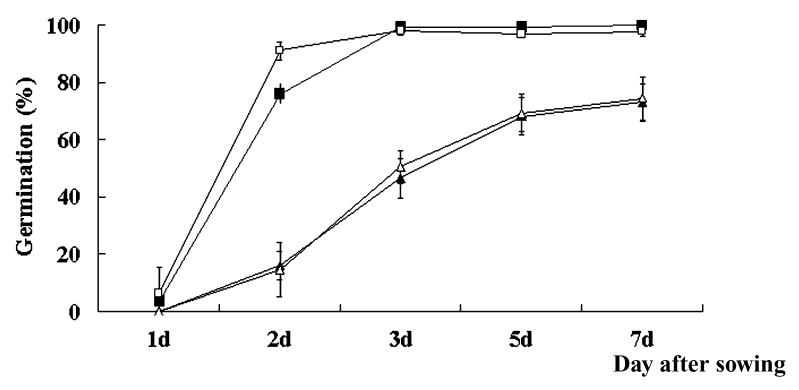

$\rightarrow-$ Ws + Suc - - Ws -Suc $\star H D A 6 / 19:$ RNAi-1 +Suc $\downarrow$ HDA6/19:RNAi-1 -Suc

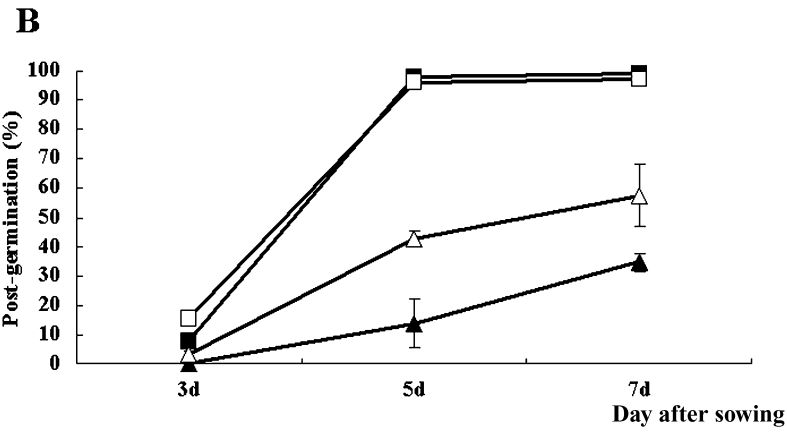

$\rightarrow-W s+$ uc $\square-$ Ws -S uc $\rightarrow$ HDA6/19:RNAi-1 +Suc - HDA6/19:RNAi-1 -Suc

Figure 2. Effect of $90 \mathrm{mM}$ sucrose on germination rate (A) and postgermination growth (B) in wild-type (ecotype Wassilewskija, Ws) and HDA6/19 double repression (HDA6/19:RNAi) seeds. Closed squares, Ws seeds sown in sucrose-containing medium; open squares, Ws seeds sown in sucrose-free medium; closed triangles, HDA6/19:RNAi-1 seeds sown in sucrose-containing medium; open triangles, HDA6/ 19:RNAi-1 seeds sown in sucrose-free medium. The values represent the mean value \pm standard deviation from three experiments.

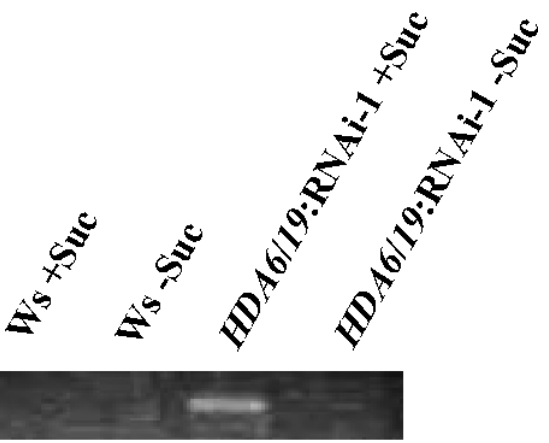

$V S P 2$

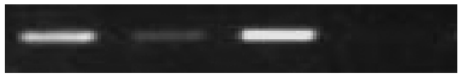

UBQ10

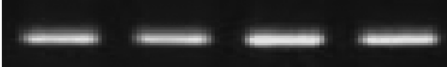

Figure 3. Effect of $90 \mathrm{mM}$ sucrose on LEC1 and VSP2 expression in wild-type (ecotype Wassilewskija, Ws) and $H D A 6 / 19$ double repression (HDA6/19:RNAi, CS24039/CS30925) seeds germinated in sucrosecontaining $(+\mathrm{Suc})$ or sucrose-free $(-\mathrm{Suc})$ medium. UBQ10 was used as an internal standard. 


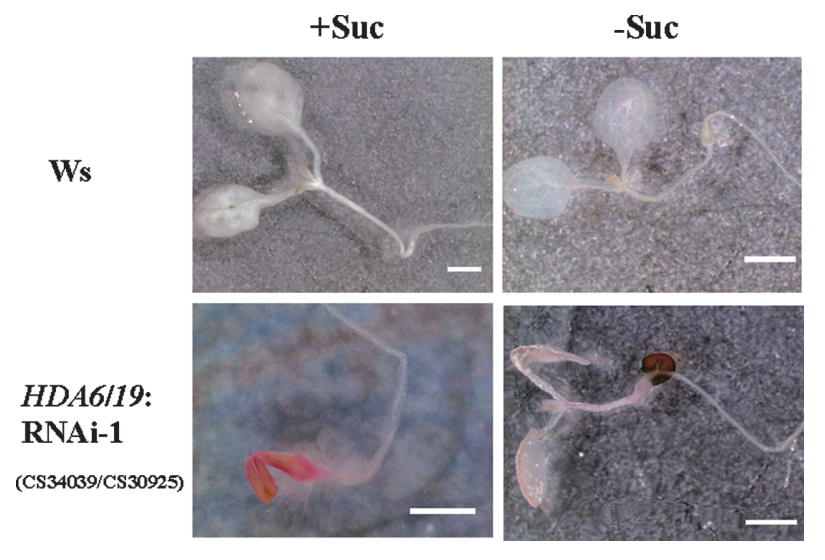

Figure 4. Fat red 7B staining in wild-type (ecotype Wassilewskija, Ws) and HDA6/19 double repression (HDA6/19:RNAi, CS24039/ CS30925) seeds germinated in sucrose-containing (+Suc) or sucrosefree $(-$ Suc $)$ medium 7 days after sowing. Bar $=1 \mathrm{~mm}$.

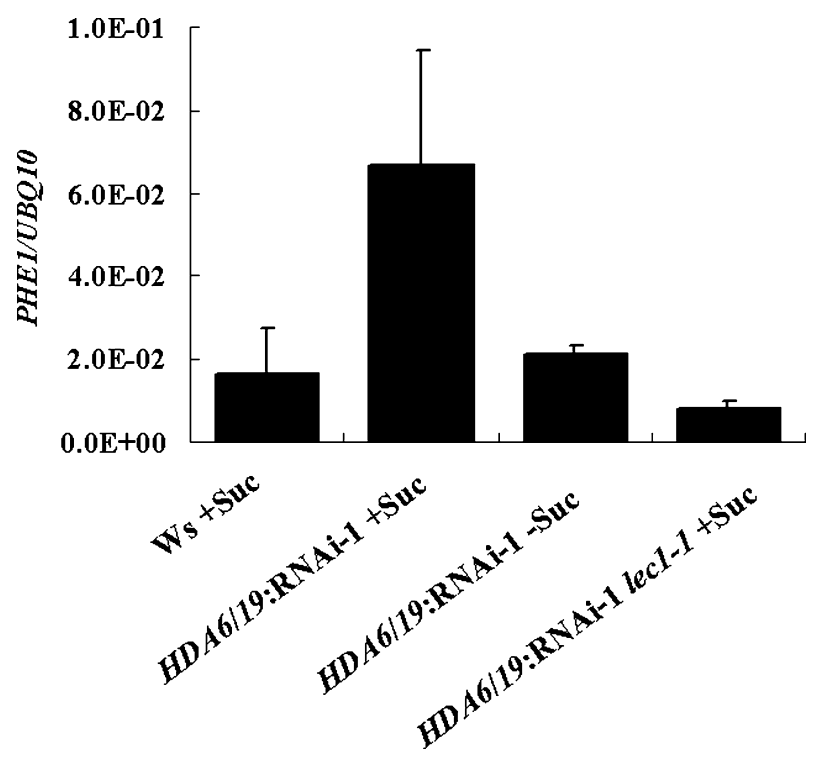

Figure 5. Effect of $90 \mathrm{mM}$ sucrose on PHE1 expression in germinated seeds. $P H E$ lexpression was analyzed 7 days after sowing via quantitative RT-PCR. The vertical lines represent $P H E 1$ expression normalized against $U B Q 10$ expression. The values represent the mean \pm standard deviation from three experiments. Ws, wild-type (ecotype Wassilewskija) seeds; HDA6/19:RNAi-1, HDA6/19 double repression line; HDA6/19:RNAi-1 lec1-1, HDA6/HDA19 double-repression line with an additional lecl mutation; + Suc, seeds germinated in sucrosecontaining medium; -Suc, seeds germinated in sucrose-free medium.

MADS-box transcription factor. The ectopic expression of PHEl and LECl occurs in $p k l$ mutants, which retain embryonic characteristics in the root even after germination ( $\mathrm{Li}$ et al. 2005). PHE1 expression was examined via quantitative RT-PCR in wild-type and HDA6/19:RNAi seeds sown in medium with or without $90 \mathrm{mM}$ sucrose (Figure 5). In wild-type plants, PHE1 expression was not observed in seedlings germinated in either medium. In contrast, high levels of PHE1 expression were observed in HDA6/19:RNAi seeds germinated in sucrose-containing medium; however,
$\mathbf{A}$
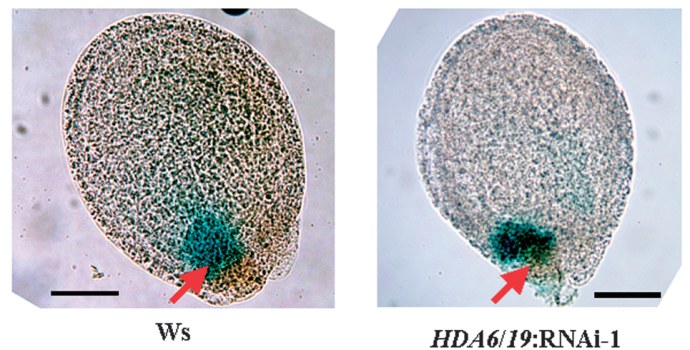

HDA6/19:RNAi-1 (CS24039/CS30925)

B
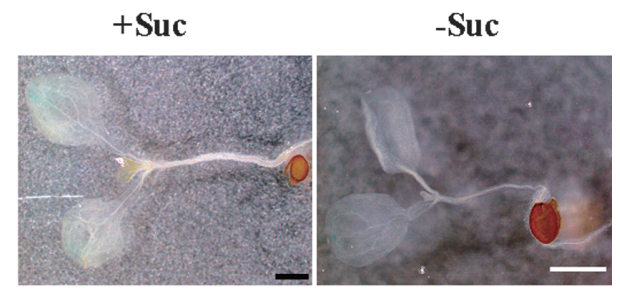

HDA6/19:

RNAi-1

(CS24039/CS30925)
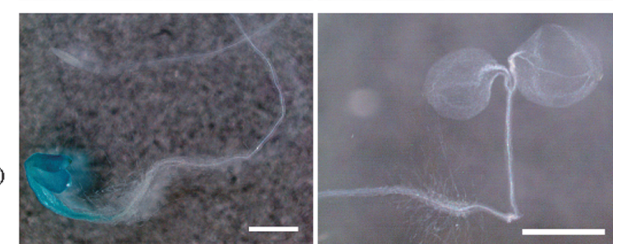

Figure 6. Localization of PHE1 expression in seeds and germinated seedlings. (A) GUS staining in wild-type (ecotype Wassilewskija, Ws) and HDA6/19:RNAi-1 (CS24039/CS30925) seeds transformed with the PHE::GUS transgene. Arrows indicate the chalazal endosperm. Bar $=0.1 \mathrm{~mm}$. (B) GUS staining in Ws and HDA6/19:RNAi-1 seedlings germinated in sucrose-containing (+Suc) or sucrose-free $(-\mathrm{Suc})$ medium. Ws and HDA6/19:RNAi-1 seeds were transformed with the $P H E:: G U S$ transgene and sown in B5 solid medium with or without sucrose. GUS staining was performed 7 days after sowing. Bar $=1 \mathrm{~mm}$.

similar to $L E C 1$ expression, $P H E 1$ expression was reduced in HDA6/19:RNAi seedlings germinated in sucrose-free medium. To examine the association between the ectopic expression of PHE1 and LEC1 in HDA6/19:RNAi seedlings, PHE1 expression was analyzed in HDA6/19: RNAi-1lec1-1 seedlings germinated in sucrose-containing medium. As a result, the introgression of a lec 1 mutation abolished the previously observed increase in PHE1 expression in HDA6/19:RNAi seedlings.

The specific expression of PHE1 in gametophytes and developing embryos is regulated by MEDEA (MEA) via histone methylation, and the ectopic expression of this factor has been observed in the endosperm of mea mutants (Köhler et al. 2003; Makarevich et al. 2006). To determine whether HDA6 and HDA19 contribute to the regulation of $P H E 1$ expression during embryogenesis, the promoter sequence of the PHE1 gene was ligated upstream of the $\beta$-glucuronidase reporter gene to create a PHE 1::GUS construct. This construct was then introduced into wild-type and HDA6/19:RNAi plants. PHE1 is specifically expressed in gametophytes and developing embryos (Köhler et al. 2003). In wild-type 
plants, GUS staining was observed in developing seeds in the chalazal endosperm (Figure 6A), which is consistent with previous reports (Köhler et al. 2003, 2005). Furthermore, HDA6/19:RNAi seeds showed a similar staining pattern to that of wild-type seeds. After germination, however, GUS staining was not observed in wild-type seedlings germinated in sucrose-containing or sucrose-free medium (Figure 6B). In the case of HDA6/ 19:RNAi seeds, GUS staining was observed in growtharrested seeds that had been germinated in sucrosecontaining medium, but not in seedlings germinated in sucrose-free medium (Figure 6B). Similar to fat red 7B staining, GUS staining was particularly intense in the closed cotyledon and the hypocotyl, but not in the growing root (Figure 6B).

\section{Discussion}

The overexpression of LECl in seedlings causes growth arrest after germination (Lotan et al. 1998). Furthermore, ectopic LEC1 expression in seedlings alters hypocotyl structure and induces the ectopic accumulation of starch and lipids, which is further enhanced in the presence of exogenous auxin and sugars, but not in the presence of $\mathrm{GA}_{3}$ or ABA (Casson and Lindsey 2006). Sugars are important regulators of germination and seedling development (Gibson et al. 2005). In addition, sugar signaling may be involved in the repression of embryonic properties after germination (Tsukagoshi et al. 2007). Based on these previous studies, sugar signaling is thought to be involved in the expression of embryospecific transcription factors and the observed postgermination growth arrest in the double repression HDA6/19:RNAi line. In the present study, the presence of $90 \mathrm{mM}$ sucrose did not affect germination (radicle emergence) in HDA6/19:RNAi seeds, but did have a negative effect on cotyledon expansion and greening. These arrested seeds were partially rescued when they were germinated in sucrose-free medium (Figures 1 and 2 ). Because the presence or absence of sugar did not affect the expression profile of the sugar-inducible gene VSP2 in HDA6/19:RNAi seeds (Figure 3), the repression of $H D A 6$ and $H D A 19$ may not affect sugar signaling.

LEC1 expression in HDA6/19:RNAi seeds was also dependent upon the presence of sucrose during germination (Figure 3). Fat red 7B staining, which indicates the presence of large amounts of triacylglycerol and the maintenance of embryonic properties (Henderson et al. 2004), was observed in the closed cotyledon and hypocotyl of HDA6/19:RNAi seeds germinated in sucrose-containing medium. However, the staining intensity was much weaker when the seeds were germinated in sucrose-free medium (Figure 4). These results suggest that both the expression of $L E C 1$ and the maintenance of embryonic properties during post- germination growth are dependent upon the presence of sugar during germination. This notion is supported by the observed increase in PHEl expression in HDA6/ 19:RNAi seedlings germinated in sucrose-containing medium (Figures 5 and 6). Growth-arrested HDA6/ 19:RNAi seeds germinated in sucrose-containing medium showed high PHE1 ::GUS transgene expression; however, GUS staining decreased when seeds were germinated in sucrose-free medium (Figure 6B). In HDA6/19:RNAi seeds germinated in sucrose-containing medium, PHE1::GUS expression and fat red 7B staining were observed in the closed cotyledon and the hypocotyl, but not in the growing root (Figures 4 and 6B); growth arrest was observed in the cotyledon, but not in the root (Figure 1C). These results are consistent with a previous report (Figure 7D and Supplemental Figure 4D in Tanaka et al. 2008). Taken together, these results suggest that post-germination growth is associated with the ectopic expression of embryonic properties.

PHE1 encodes a MADS-box transcription factor that shows transient expression during early embryogenesis (Köhler et al. 2003). Embryo-specific expression of PHE1 is directly regulated by MEA, a member of the polycomb group proteins, which mediates histone methylation (Köhler et al. 2003; Makarevich et al. 2006). Because histone methylation is occasionally coupled with histonedeacetylation (Peterson and Laniel 2004), and because PHE1 is ectopically expressed in the endosperm and induces seed abortion in mea mutants (Köhler et al. 2003), the misregulation of PHE1 may also occur in the endosperm of HDA6/19:RNAi seeds. However, we did not observe the expression of the PHE1::GUS transgene in the endosperm of HDA6/ 19:RNAi seeds (Figure 6A). In addition, PHE1 expression in germinated HDA6/19:RNAi seedlings was three times higher than that in wild-type seedlings germinated in sucrose-containing medium; however, PHE1 expression disappeared when HDA6/19:RNAi seeds were sown in sucrose-free medium (Figure 5).

Previous studies showed that the ectopic expression of PHEland LECl occurred in pkl mutants that retained embryonic characteristics in the root even after germination ( $\mathrm{Li}$ et al. 2005). In the present study, the introgression of a lec 1 mutation abolished the previously observed increase in PHE1 expression in HDA6/ 19:RNAi seeds germinated in sucrose-containing medium. These results exclude the possibility of direct HDA6- or HDA19-mediated PHE1 inhibition via histonedeacetylation at the PHE1 locus during embryogenesis. It is more likely that this sucrosedependent PHE1 expression is triggered by the expression of embryo-specific transcriptional factors. In conclusion, our results indicate that HDA6 and HDA19 repress embryonic properties via the regulation of embryo-specific transcription factors. Sugar signaling 
may also contribute to this repression of embryonic properties.

\section{Acknowledgements}

This work was supported in part by Grants-in-Aid for Special Research on Priority Areas (grant-in-aid no. 19043007 to H.K.) from the Ministry of Education, Science, Culture, and Sports, Japan.

\section{References}

Brundrett MC, Kendrick B, Peterson CA (1991) Efficient lipid staining in plant material with sudan red $7 \mathrm{~B}$ or fluorol [correction of fluoral] yellow 088 in polyethylene glycolglycerol. Biotech Histochem 66: 111-116

Casson SA, Lindsey K (2006) The turnip mutant of Arabidopsis reveals that LEAFY COTYLEDON1 expression mediates the effects of auxin and sugars to promote embryonic cell identity. Plant Physiol 142: 526-541

Chen ZJ, Tian L (2007) Roles of dynamic and reversible histone acetylation in plant development and polyploidy. Biochim Biophys Acta 1769: 295-307

Dejardin A, Sokolov LN, Kleczkowski LA (1999) Sugar/ osmoticum levels modulate differential abscisic acid-independent expression of two stress-responsive sucrose synthase genes in Arabidopsis. Biochem J 344: Pt 2:503-509

Gibson SI (2005) Control of plant development and gene expression by sugar signaling. Curr Opin Plant Biol 8: 93-102

Grossniklaus U, Schneitz K (1998) The molecular and genetic basis of ovule and megagametophyte development. Semin Cell Dev Biol 9: 227-238

Henderson JT, Li HC, Rider SD, Mordhorst AP, Romero-Severson J, Cheng JC, Robey J, Sung ZR, de Vries SC, Ogas J (2004) PICKLE acts throughout the plant to repress expression of embryonic traits and may play a role in gibberellin-dependent responses. Plant Physiol 134: 995-1005

Karimi M, Inzé D, Depicker A (2002) GATEWAY vectors for Agrobacterium-mediated plant transformation. Trends Plant Sci 7: 193-195

Köhler C, Grossniklaus U (2002) Epigenetic inheritance of expression states in plant development: the role of Polycomb group proteins. Curr Opin Cell Biol 14: 773-779
Köhler C, Hennig L, Spillane C, Pien S, Gruissem W, Grossniklaus U (2003) The Polycomb-group protein MEDEA regulates seed development by controlling expression of the MADS-box gene PHERES1. Genes Dev 17: 1540-1553

Köhler C, Page DR, Gagliardini V, Grossniklaus U (2005) The Arabidopsis thaliana MEDEA Polycomb group protein controls expression of PHERES1 by parental imprinting. Nat Genet 37 : $28-30$

Li HC, Chuang K, Henderson JT, Rider SD Jr, Bai Y, Zhang H, Fountain M, Gerber J, Ogas J (2005) PICKLE acts during germination to repress expression of embryonic traits. Plant $J$ 44: $1010-1022$

Lotan T, Ohto M, Yee KM, West MA, Lo R, Kwong RW, Yamagishi K, Fischer RL, Goldberg RB, Harada JJ (1998) Arabidopsis LEAFY COTYLEDON1 is sufficient to induce embryo development in vegetative cells. Cell 93: 1195-1205

Makarevich G, Leroy O, Akinci U, Schubert D, Clarenz O, Goodrich J, Grossniklaus U, Kohler C (2006) Different Polycomb group complexes regulate common target genes in Arabidopsis. EMBO Rep 7: 947-952

Peterson CL, Laniel MA (2004) Histones and histone modifications. Curr Biol 14: R546-R551

Price J, Li TC, Kang SG, Na JK, Jang JC (2003) Mechanisms of Glucose Signaling during Germination of Arabidopsis. Plant Physiol 132: 1424-1438

Suzuki M, Wang HH-Y, McCarty DR (2007) Repression of the LEAFY COTYLEDON 1/B3 regulatory network in plant embryo development by VP1/ABSCISIC ACID INSENSITIVE 3-LIKE B3 genes. Plant Physiol 143: 902-911

Tanaka M, Kikuchi A, Kamada H (2008) The Arabidopsis histone deacetylases HDA6 and HDA19 contribute to the repression of embryonic properties after germination. Plant Physiol 146: 149-161

To A, Valon C, Savino G, Guilleminot J, Devic M, Giraudat J, Parcy F (2006) A network of local and redundant gene regulation governs Arabidopsis seed maturation. Plant Cell 18: 1642-1651

Tsukagoshi H, Morikami A, Nakamura K (2007) Two B3 domain transcription repressors prevent sugar-inducible expression of seed maturation genes in Arabidopsis seedlings. Proc Natl Acad Sci USA 104: 2543-2547

Wanger D (2003) Chormatin regultion of plant development. Curr Opin Plant Biol 6: 20-28 